\title{
Labeling of DOTA-conjugated HPMA-based polymers with trivalent metallic radionuclides for molecular imaging
}

\author{
Elisabeth Eppard ${ }^{1}$, Ana de la Fuente ${ }^{2}$, Nicole Mohr ${ }^{3}$, Mareli Allmeroth ${ }^{3}$, Rudolf Zentel ${ }^{3}$, Matthias Miederer ${ }^{4}$,
} Stefanie Pektor ${ }^{4^{*}}$ and Frank Rösch ${ }^{2}$

\begin{abstract}
Background: In this work, the in vitro and in vivo stabilities and the pharmacology of HPMA-made homopolymers were studied by means of radiometal-labeled derivatives. Aiming to identify the fewer amount and the optimal DOTA-linker structure that provides quantitative labeling yields, diverse DOTA-linker systems were conjugated in different amounts to HPMA homopolymers to coordinate trivalent radiometals Me(III)* = gallium-68, scandium-44, and lutetium-177.
\end{abstract}

Results: Short linkers and as low as 1.6\% DOTA were enough to obtain labeling yields $>90 \%$. Alkoxy linkers generally exhibited lower labeling yields than alkane analogues despite of similar chain length and DOTA incorporation rate. High stability of the radiolabel in all examined solutions was observed for all conjugates. Labeling with scandium-44 allowed for in vivo PET imaging and ex vivo measurements of organ distribution for up to $24 \mathrm{~h}$.

Conclusions: This study confirms the principle applicability of DOTA-HPMA conjugates for labeling with different trivalent metallic radionuclides allowing for diagnosis and therapy.

Keywords: DOTA-HPMA conjugates, Theranostic, Radiolabeling, Gallium-68, Scandium-44, Lutetium-177, PET, Biodistribution

\section{Background}

One of the major problems in current chemotherapy is the lack of selectivity of the utilized anticancer drugs. Conventional chemotherapeutic drugs suffer from a narrow therapeutic index as a consequence of affecting not only cancer cells but basically all cells with high proliferation rates. The therapeutic index of a drug can be improved by increasing the accumulation at the target site, e.g., the tumor $[1,2]$. Nanomaterials $(<100 \mathrm{~nm})$ can often improve pharmacokinetics due to their particle size, charge, and shape and may passively and/or actively accumulate in tumor tissue. Since the early 1970s, $N$-(2-hydroxypropyl)methacrylamide (HPMA) is investigated as drug delivery system based on the

\footnotetext{
* Correspondence: Stefanie.pektor@unimedizin-mainz.de

E. Eppard and A. de la Fuente contributed equally to the first authorship.

S. Pektor and F. Rösch contributed equally to the last authorship.

${ }^{4}$ Clinic for Nuclear Medicine, University Medical Center Mainz,

Langenbeckstraße 1, 55131 Mainz, Germany

Full list of author information is available at the end of the article
}

enhanced permeability and retention (EPR) effect [3-5]. HPMA-based copolymers are hydrophilic, biocompatible, and non-immunogenic [6,7], and selection as drug carrier is based on detailed studies of the relationship between structure and biocompatibility of hydrophilic polymers [8-13]. HPMA-copolymer conjugates have been successfully utilized in the delivery of miscellaneous drugs (e.g., doxorubicin [14], paclitaxel [15], camptothecin [16]). Modification can be achieved through activated comonomers leading to biocompatible polymers with functionalities for polymer analogue reaction. This strategy is very flexible in the choice of ligands, type of activation, and occupancy rate and was already used in various studies [17].

As it was shown before, nanomaterials like HPMAbased nanocarriers are able to combine both imaging agents and drugs within one formulation $[8,18,19]$ for the in vivo analysis by positron emission tomography (PET) [20, 21]. Those studies include information about 
the early pharmacology of fluorine-18-radiolabelled HPMA derivatives, yielding information of excretion pathways, blood retention, and organ distribution. According to the physical half-life of fluorine-18 of $110 \mathrm{~min}$, those data only cover a period of 2 to $4 \mathrm{~h}$ post injection. For an investigation of later time points, in particular when drug accumulation in tumors is of interest, radionuclides of longer physical half-life are needed. Here, we intent to introduce a radiolabeling concept based on a chelator being (i) adequate to the coordination chemistry of trivalent metals, namely DOTA (1,4,7,10-tetraazacyclododecane-1,4,7,10tetraacetic acid), and being (ii) covalently attachable to the HPMA structure.

Two generator-produced positron emitters of choice are gallium- 68 and scandium-44, which can be applied to quantitative in vivo imaging via PET. Gallium-68 is a positron emitter (89\%) with a half-life of $67.71 \mathrm{~min}$ and average positron energy of $1.899 \mathrm{MeV}$. Its physical halflife is adequate to allow the preparation and purification of gallium-68-labeled radiopharmaceuticals and for imaging biological processes with short half-life. The major advantage of gallium-68 is its availability via a ${ }^{68} \mathrm{Ge} /$ ${ }^{68} \mathrm{Ga}$-generator system, which provides a cyclotronindependent and cost-effective source of the isotope in suitable conditions for labeling after its post-processing [22-27]. Scandium-44 (94\% positron emission) is available via the ${ }^{44} \mathrm{Ti} /{ }^{44} \mathrm{Sc}$-generator system $[28,29]$. Due to its longer physical half-life of $3.97 \mathrm{~h}$, scandium-44 facilitates the tracking of processes with longer biological half-life than gallium-68 or even fluorine-18. It could be applied for more exact planning and dosimetry calculations in endoradiotherapy [27]. In addition, longer-lived lutetium-177 (6.7 days) can be used as well for ex vivo organ distribution studies. Lutetium-177 is not suitable for PET imaging, but as a result of emitting gamma radiation $(113.0$ and $208.4 \mathrm{keV})$ in addition to its $\beta^{-}$particles, it can be used for therapy and SPECT imaging [30]. Those radiolabeled HPMA derivatives appear also to be suitable to investigate processes such as the EPRmediated accumulation of nanoparticles in tumor tissue.

In addition, HPMA polymers could also be labeled with iodine-123 [31] to visualize tumors and metastases by SPECT or $99 \mathrm{~m}$-technetium [32] as it was done before. To our knowledge so far, no studies exist with iodine-124-labeled HPMA polymers but this radionuclide was already used to enable long-term tumor PET imaging of clinically approved silica nanoparticles [33] and could also be a useful tool for imaging longcirculating HPMA polymers.

In the present study, we aimed to synthesize DOTAconjugated HPMA homopolymers with alkyl- and alkoxy linkers for labeling with trivalent metallic radionuclides. The main objective was to show how the linker (in terms of structure and length) as well as the amount of incorporated DOTA may influence labeling kinetics, stability, and whether labeling procedures for different radionuclides can be utilized. Labeling of the synthesized DOTA-conjugated homopolymers was first performed with gallium-68 to evaluate reaction parameters (incubation time, amount of compound, temperature, influence of organic solvent). The stability of the gallium-68labeled conjugates was analyzed in vitro in $0.9 \% \mathrm{NaCl}$ and human serum albumin (HSA), as well as in the presence of different metal cations and other competing chelators, like EDTA and DTPA. Additionally, labeling with the diagnostic and therapeutic isotopes scandium44 and lutetium-177 was performed to allow for the characterization of late organ distribution profiles of the HPMA-based nanoparticles in following studies. Proof of principle in vivo distribution was investigated by PET with a HPMA-DOTA conjugate of $13 \mathrm{kDa}$, a short ethyl linker, and a low DOTA/HPMA ratio (1.6\%), which was radiolabeled with scandium- 44 .

\section{Methods}

All chemicals were analytical or pure reagent grade and used as received unless otherwise specified. DOTA$\left({ }^{\mathrm{t}} \mathrm{Bu}\right)_{3}$ was obtained from CheMatech (Dijon, France). All other organic and inorganic reagents were purchased from Sigma-Aldrich (Munich, Germany) and Across Organics (Geel, Belgium). Deionized Milli-Q water (18.2 $\mathrm{M} \Omega \mathrm{cm}$; Millipore, Darmstadt, Germany) was used in all organic reactions. Dioxane was distilled over a sodium/potassium composition. Lauryl methacrylate was distilled to remove the stabilizer and stored at $-18{ }^{\circ} \mathrm{C}$. 2,2' -Azo-bis-(isobutyronitrile) (AIBN) was recrystallized from diethyl ether and stored at $-18{ }^{\circ} \mathrm{C}$ as well. ${ }^{1} \mathrm{H}$ NMR spectra were obtained by a Bruker AC 300 spectrometer (Bruker, Karlsruhe, Germany) at $300 \mathrm{MHz}$. The amount of incorporated DOTA derivatives 17-21 in the polymers was determined by inverse gated ${ }^{13} \mathrm{C}-\mathrm{NMR}$. ${ }^{19}$ F-NMR analysis was carried out with a Bruker DRX400 at $400 \mathrm{MHz}$. It proved the complete conversion of the reactive ester units by reaction with the DOTA derivatives and 2-hydroxy-propylamine in analogy to [34]. The synthesized polymers were dried at $40{ }^{\circ} \mathrm{C}$ under vacuum overnight, followed by gel permeation chromatography (GPC). GPC was performed in tetrahydrofuran (THF) as solvent, using the following equipment: pump PU 1580, autosampler AS 1555, UV detector UV 1575, and RI detector RI 1530 from Jasco (Pfungstadt, Germany) as well as a miniDAWN Tristar light scattering detector from Wyatt (Dernbach, Germany). Columns were used from MZ Analysentechnik, $300 \times 8.0 \mathrm{~mm}$ : MZ-Gel SDplus $106 \AA 5 \mu \mathrm{m}$, MZ-Gel SDplus $104 \AA 5 \mu \mathrm{m}$, and MZ-Gel SDplus $102 \AA 5 \mu \mathrm{m}$. GPC data were evaluated by using the software PSS WinGPC Unity (Polymer Standard Service Mainz, Germany). The flow rate was set to $1 \mathrm{~mL} / \mathrm{min}$ with 
a temperature of $25{ }^{\circ} \mathrm{C}$. Commercial ${ }^{68} \mathrm{Ge} /{ }^{68} \mathrm{Ga}$ generators based on $\mathrm{TiO}_{2}$ phase absorbing ${ }^{68} \mathrm{Ge}(\mathrm{IV})$ were obtained from Cyclotron Co., Ltd. (Obninsk, Russia). Typically, batch activities of $80-150 \mathrm{MBq}$ were used. Scandium-44 was provided from a ${ }^{44} \mathrm{Ti} /{ }^{44} \mathrm{Sc}$-generator system developed in Mainz [28]. Batch activities of 165-180 MBq were used. Lutetium-177 was obtained from ITG (Munich, Germany) and used without further purification. Counting was performed in a borehole counter (Nuklear-Medizintechnik Dresden $\mathrm{GmbH}$, Germany). Thin-layer chromatography (TLC) was performed on silica-gel (silica-gel 60F254; MERCK, Darmstadt, Germany)-coated aluminum TLC sheets and analyzed using an instant imager (Instant Imager, Canberra Packard, Schwadorf, Austria). The cation exchange resins AG 50W-X8 (- 400 mesh), AG 50W-X4 (200-400 mesh), and AG $50 \mathrm{~W}-\mathrm{X} 8$ (200-400 mesh) were obtained from Bio-Rad (Munich, Germany). Strata-X mini-C-18 cartridges were obtained from Phenomenex (Aschaffenburg, Germany). HiTrap ${ }^{\text {тs }}$ Desalting Columns were purchased from GE-Healthcare Europe $\mathrm{GmbH}$ (Freiburg, Germany). TraceSelect water (Sigma-Aldrich, Germany) was used for all aqueous radiolabeling solutions.

Synthesis and characterization of DOTA-HPMA conjugates The DOTA-HPMA conjugates were prepared following the synthesis routes depicted in Figs. 1 and 2. Figure 1 shows the preparation of different DOTA linkers that later on were conjugated to the HPMA backbones in different amounts. Details on the synthesis route and characterization are added in the supplementary information. The hydrodynamic radius ( $\mathrm{Rh}$ ) of the polymers $(0.1 \mathrm{mg} / \mathrm{ml}$ in isotonic $\mathrm{NaCl}$ solution) was determined by Fluorescence Correlation Spectroscopy (FCS) at RT.

\section{Radiochemistry}

Labeling with gallium-68 Labeling of DOTA-conjugated HPMA homopolymers was performed by mixing aliquots of polymer stock solution (1 $\mathrm{mg} / \mathrm{mL}$ in water) with postprocessed gallium-68 eluate $[24,25]$. Investigation of the amount of conjugate needed for labeling reaction was performed by varying time and concentration of DOTAconjugated polymer using conjugate 31 with acetone postprocessed gallium-68 and was adopted for all the other labeling reactions. In addition, labeling with HPMA homopolymer without DOTA functionalization was performed under these conditions to investigate the unspecific binding. Reaction mixtures were heated up to $95^{\circ} \mathrm{C}$ for $30 \mathrm{~min}$.

Labeling with scandium-44 Labeling of DOTAconjugated HPMA homopolymers was performed with $250 \mu \mathrm{L}$ polymer stock solution $(1 \mathrm{mg} / \mathrm{mL}$ in water) in post-processed scandium-44 eluate $(3 \mathrm{~mL})$ and heating up to $95{ }^{\circ} \mathrm{C}$ for $30 \mathrm{~min}$ [35].

Labeling with lutetium-177 Labeling of DOTAconjugated HPMA homopolymers was performed with $250 \mu \mathrm{L}$ polymer stock solution $(1 \mathrm{mg} / \mathrm{mL}$ in water $)$ with $150 \mu \mathrm{L} 0.1 \mathrm{M} \mathrm{Na}$ acetate buffer $(\mathrm{pH}=8)$ and $100 \mu \mathrm{L}$ lutetium-177 and heating up to $95{ }^{\circ} \mathrm{C}$ for $30 \mathrm{~min}$. The stoichiometric ratio polymer-to-lutetium-177 was 10:1.

Purification of the labeled conjugates The radiolabeled polymeric systems were cleaned from unreacted radiometal by Sephadex G-25 size exclusion chromatography (HiTrap ${ }^{\text {тм }}$ Desalting Column, Sephadex G-25 Superfine, $0.9 \% \mathrm{NaCl}$, flow rate: $0.5 \mathrm{~mL} / \mathrm{min}$ ) leading to a pure, gallium-68-labeled polymer solution ready for subsequent experiments.

Quality control Quality control of complex formation was performed by radio thin-layer chromatography (radio-TLC). The TLC plates were developed with $0.1 \mathrm{M}$ citric buffer $(\mathrm{pH}=4)$. Quantitative distribution of radioactivity on TLC plates was measured using an instant imager.

Stability of the labeled compounds Stability studies were performed with gallium-68-labeled polymers of radiochemical purity $>98 \%$ in physiological conditions and in the presence of competing metals and chelates.

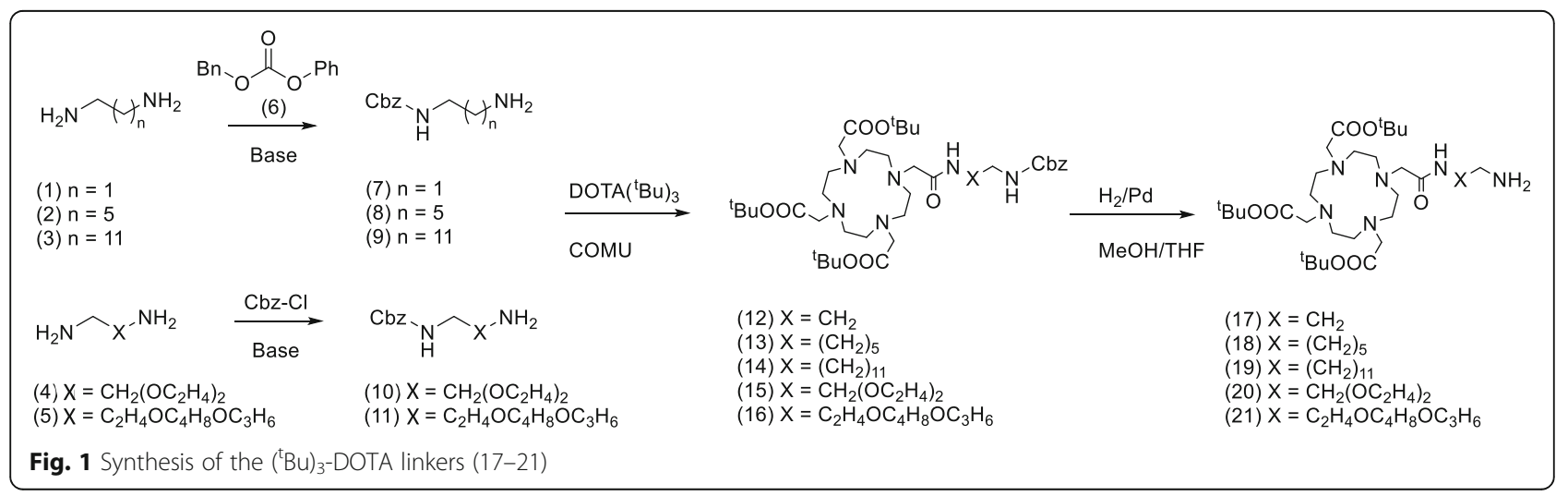




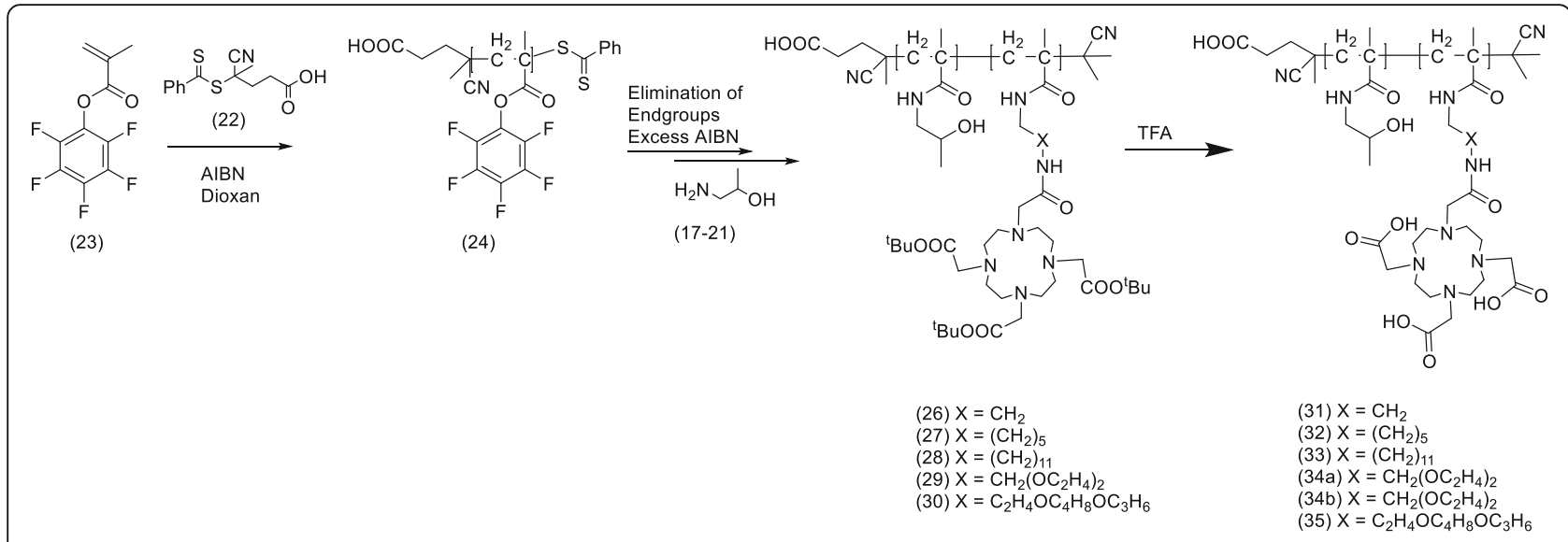

Fig. 2 Synthesis of the HPMA-linker-DOTA systems (31-35)

Detailed setup is explained in the supplementary section. As nonspecific binding of the metal cation to nanoparticles is a basic problem in labeling those systems, investigation of nonspecific binding of gallium-68 to a HPMA polymer was performed with 20 nmol HPMA homopolymer without functionalization (Mn: $12.000 \mathrm{~g} / \mathrm{mol}$, 0\% DOTA) utilizing the optimized protocol.

\section{In vivo $\mathrm{PET}$ imaging and ex vivo biodistribution}

Six-8-week-old male C57BL/6J (Janvier, France) mice were housed under specific pathogen-free conditions in the animal care facility in Mainz according to the guidelines of the regional animal care committee. All experiments were performed in accordance with federal guidelines and approved by the ethical committee of the state of Rheinland-Pfalz (according to $\$ 8$ Abs. 1 Tierschutzgesetz, Landesuntersuchungsamt; permission no. 23177-07/G121-092 to M. Miederer). All injections and imaging experiments were done under isoflurane anesthesia to minimize suffering. Mice were injected subcutaneously with $2 \times 10^{5}$ murine B16-F10 melanoma tumor cells into the right flank. When tumors reached a volume of $150-200 \mathrm{~mm}^{3},{ }^{44} \mathrm{Sc}$-labeled polymer conjugate $31 \mathrm{c}(1.5 \mathrm{mg}$ in $1 \mathrm{~mL}$ of isotonic saline) was injected i.v. in anesthetized mice with a mean activity of $4.6 \pm$ $0.5 \mathrm{MBq}$. Whole body scandium-44 PET scans were dynamically acquired over 120 min post injection (p.i.), or 30 - min static scans were performed $90 \mathrm{~min}, 210 \mathrm{~min}$, and $24 \mathrm{~h}$ p.i.

All PET imaging studies were performed on a nanoScan PET/MRI (Mediso, Budapest, Hungary), and PET data were dynamically reconstructed with Teratomo 3D (4 iterations, 6 subsets, voxel size $0.4 \mathrm{~mm}$ ) using user defined frames $(3 \times 20 ; 3 \times 60 ; 3 \times 120 ; 10 \times$ $300 ; 6 \times 600 \mathrm{~s})$ or statically using one 30 -min frame. Afterwards, the PET images were analyzed with pmod software (version 3.6).
After 2, 4, and $24 \mathrm{~h}$, the animals were sacrificed and different organs and blood were removed. The tissue samples were weighed and the ${ }^{44} \mathrm{Sc}$ activity in the organs was directly measured in a $\gamma$-counter (2470 WIZARD2 Automatic Gamma Counter, PerkinElmer) to calculate the percentage of accumulated activity as $\% \mathrm{ID} / \mathrm{g}$.

\section{Ex vivo metabolism evaluation}

Blood samples (ca. $100 \mu \mathrm{l}$ ), collected during the biodistribution, were mixed with heparin solution $(150 \mu \mathrm{l})$ to avoid coagulation. Blood samples were weighed and activity was measured in a $\gamma$-counter; $500 \mu \mathrm{l}$ of PBS were added and the blood was centrifuged at $1700 \times g$ for $3 \mathrm{~min}$ in order to separate blood cells and plasma. The plasma fractions were weighed and activity was measured in a $\gamma$-counter. Proteins and polymers were precipitated by adding the same volume of acetonitrile to the plasma fraction. To separate proteins and polymer from plasma water, the samples were centrifuged at $1700 \times g$ for $3 \mathrm{~min}$. The supernatants were weighed followed by determination of the activity in a $\gamma$-counter. The percentage of radioactivity in the blood cells, protein plus polymers, and plasma water fractions was calculated by subtracting the activities of the supernatants from the activity of the whole blood sample.

\section{Results}

\section{Organic synthesis}

For coupling reaction between the HPMA backbone and the bifunctional chelator, orthogonal amidation chemistry via active ester groups was selected [17]. Synthesis of the amine-terminated DOTA-based ligands 17-21 from commercially available $\left({ }^{\mathrm{t}} \mathrm{Bu}\right)_{3}$-DOTA is depicted in Fig. 1.

First, the commercially available $\alpha, \omega$-diamines 1-5 were mono-Cbz-protected. The protection was realized with benzylphenylcarbonate $\mathbf{6}$ for 1-3 (Additional file 1) 
and with benzylchloroformate for 4-5. The selectivity of the mono-Cbz-protection using $\mathbf{6}$ decreases with the length of the alkyl chain but achieves good yields for 1-3. Mono-Cbz-protection with $\mathbf{6}$ was ineffective for the alkoxy diamines 4-5 so benzylchloroformate was used instead obtaining good yields of the desired product. The following amidation of $\left({ }^{\mathrm{t}} \mathrm{Bu}\right)_{3}$-DOTA with 7-11 afforded the Cbz-protected ( $\left.{ }^{\mathrm{t}} \mathrm{Bu}\right)_{3}$-DOTA ligands $\mathbf{1 2 - 1 6}$ in good yields of $66-79 \%$. The Cbz-protecting group was removed via hydrogenation to obtain the $\left({ }^{\mathrm{t}} \mathrm{Bu}\right)_{3}$-DOTA ligands 17-21 in almost quantitative yields (93-97\%).

The synthetic route to the DOTA-HPMA conjugates is depicted in Fig. 2. Starting from pentafluorophenyl methacrylate monomer $\mathbf{2 3}$, the precursor homopolymer $\mathbf{2 4}$ was synthesized according to literature (Additional file 1). The dithioester end group was removed by an excess of 2,2'-azo-bis-(isobutyronitrile) as described in literature (Additional file 1) taking the advantage of circumventing side reactions during the next reaction steps. Functionalization of the polymeric precursor was obtained by aminolysis via the amine-terminated $\left({ }^{\mathrm{t}} \mathrm{Bu}\right)_{3}$-DOTA ligands 17-21. The incorporation was calculated to be between $0.6-11 \%$ DOTA for each conjugate by inverse gated ${ }^{13} \mathrm{C}$-NMR spectroscopy. Addition of 2-hydroxypropylamine leads to the polymeric structures 26-30. The entire conversion of the reactive ester groups was proved by means of ${ }^{19} \mathrm{~F}$-NMR spectroscopy, which demonstrates complete disappearance of ${ }^{19} \mathrm{~F}$ signals of the polymeric backbone in analogy to [34]. As these reactions were performed in waterfree conditions, they happen without side reactions [34]. In a final step, the chelator was deprotected using TFA. The pure HPMA-DOTA conjugates, ready for labeling, could be obtained as white powders with good yields of $89 \%$, except conjugate 32, which could not be recovered from the dialysis flexible tube.

As intended, five different DOTA-conjugated polymer conjugates with relatively low polydispersity index (PDI) and narrow molecular weight distribution $\left(R_{h}\right.$ : 1.3$1.5 \mathrm{~nm}$ ) have been achieved by RAFT polymerization and successfully functionalized in a following polymer analogous reaction. Their molecular weight average

Table 1 Analytical data of the final HPMA-DOTA conjugates 31-35

\begin{tabular}{lllll}
\hline Polymer & $\mathrm{Mn}[\mathrm{g} / \mathrm{mol}]$ & $\mathrm{PDI}^{\mathrm{a}}$ & \% DOTA & Linker \\
\hline 31 & 15.000 & 1.3 & 11 & $(1)$ \\
33 & 12.000 & 1.2 & 1.6 & (3) \\
$34 \mathrm{a}$ & 12.000 & 1.2 & 0.6 & $(4)$ \\
$34 \mathrm{~b}$ & 12.000 & 1.2 & 1.2 & $(4)$ \\
35 & 13.000 & 1.2 & 3.5 & $(5)$ \\
\hline
\end{tabular}

${ }^{\mathrm{a}}$ Determined by GPC in THF as solvent

${ }^{b}$ Determined by ${ }^{1} \mathrm{H}-\mathrm{NMR}$ spectroscopy after polymer analogous reaction with 2-hydroxypropylamine numbers (Mn), polydispersity indices (PDI), and \% of incorporated chelators are shown in Table 1.

Later on, in order to have only one variable for direct comparison (the conjugates so far presented different linker structures with different incorporation rates of DOTA), conjugate 31 was modified with different percentages of DOTA, affording the conjugates 31a-31c following the same synthesis route as described for conjugate 31 (Table 2).

\section{Radiochemistry}

DOTA derivatives generally require elevated temperatures for complex formation in contrast to acyclic chelators; $95{ }^{\circ} \mathrm{C}$ was used as standard temperature for labeling experiments with the DOTA-HPMA conjugates with different radionuclides (e.g., gallium-68, scandium-44, lutetium177). Figure 3 shows the kinetics of $\left[{ }^{68} \mathrm{Ga}\right] \mathrm{Ga}-31$ complexation depending on the amount of the DOTA-HPMA 31 ranging from 12 to $36 \mathrm{nmol}$. The accessible labeling yield is not influenced by the amount of conjugate used. Only $12 \mathrm{nmol}$ showed a slightly lower labeling yield. Thereon, in order to work with the fewest amount of polymer while achieving maximal labeling yield in a short time, $20 \mathrm{nmol}$ was used for further experiments. Labeling with generator-derived and acetone post-processed gallium-68 in water is accomplished within $15 \mathrm{~min}$ at $95^{\circ} \mathrm{C}$ with $\mathrm{RCY}$ of $90 \%$. The complex formation is fast and efficient. Investigation of nonspecific binding of gallium-68 to a HPMA polymer was performed with 20 nmol HPMA homopolymer without DOTA functionalization. Within $25 \mathrm{~min}$, less than $5 \%$ of the radioactivity binds to the HPMA homopolymer (Fig. 3).

Next, it was tested if the radiolabeling yields are influenced by the DOTA incorporation rate in polymers with the same linker length (Fig. 4). It can be observed that radiolabeling kinetics is not directly proportional to the amount of incorporated chelator. The fastest radiolabeling kinetics are shown for conjugate 31a with 4.8\% DOTA. Slower kinetics are achieved for conjugate 31 with $11 \%$ DOTA and slightly slower radiolabeling kinetics for the polymer with $1.6 \%$ and with $3.3 \%$ of incorporated DOTA. After $25 \mathrm{~min}$, all conjugates reach the same labeling yield and do not show further differences.

Table 2 Analytical data of the final HPMA-DOTA conjugates (derivatives of type 31)

\begin{tabular}{lllll}
\hline Polymer & $\mathrm{Mn}[\mathrm{g} / \mathrm{mol}]$ & $\mathrm{PDI}^{\mathrm{a}}$ & \% DOTA $^{\mathrm{b}}$ & Linker \\
\hline 31 & 15.000 & 1.2 & 11 & $(1)$ \\
$31 \mathrm{a}$ & 14.000 & 1.2 & 4.8 & $(1)$ \\
$31 \mathrm{~b}$ & 13.000 & 1.2 & 3.3 & $(1)$ \\
$31 \mathrm{c}$ & 13.000 & 1.2 & 1.6 & $(1)$ \\
\hline
\end{tabular}

${ }^{\mathrm{a}}$ Determined by GPC in THF as solvent

${ }^{b}$ Determined by ${ }^{1} \mathrm{H}$-NMR spectroscopy after polymer analogous reaction with 2-hydroxypropylamine 


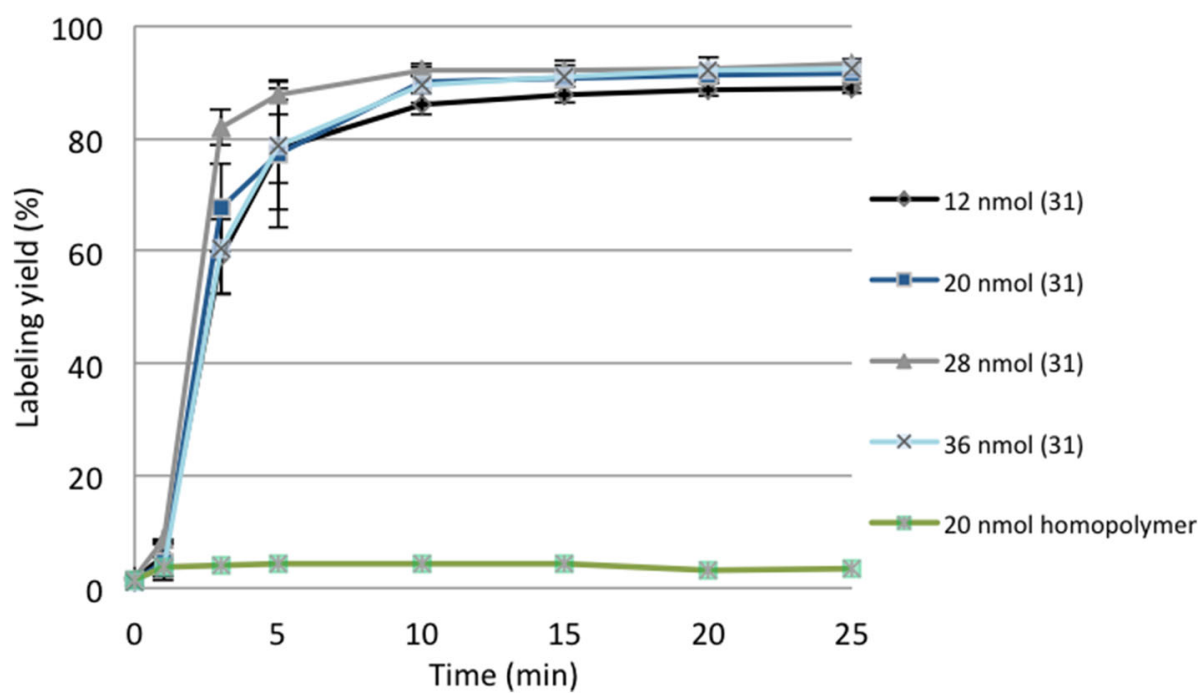

Fig. 3 Time course of ${ }^{68} \mathrm{Ga}$-complex formation with varying amounts of DOTA-HPMA conjugate 31 and polymer (20 nmol) not modified with DOTA as a control

One could hypothesize that labeling efficiency will be related to linker length and linker structure. To test this, DOTA-HPMA conjugates $\mathbf{3 1}, \mathbf{3 3}, \mathbf{3 4 a} \mathbf{a} \mathbf{b}$, and 35 were labeled using the same conditions as before. Within 15 min, labeling yields of 44-92\% can be achieved (Fig. 5, Table 3). The highest labelling yields can be found for conjugate 31 with the shortest alkyl linker (91\%) and highest DOTA content (11\%) followed by conjugate 33 with the longest alkyl linker (75\%) and 10-fold lower DOTA content $(1.6 \%)$. This shows that labeling yield is affected by the length of the linker as well as the DOTA content. Furthermore, all conjugates with alkoxy linkers showed distinct lower radiolabeling yields compared to those with alkyl linkers whereupon conjugate 34b shows slightly higher labelling yield than conjugate $\mathbf{3 5}$ with the longer alkoxy linker showing again the influence of the linker length. Conjugate 34a with a very low DOTA content $(0.6 \%)$ shows almost no labeling.

Recently, the benefit of additional use of organic solvents to facilitate radiolabeling was proven [36] and utilized in an ethanol-based post-processing. It has been shown that this ethanol-based post-processing protocol significantly increases radiochemical yields even at lower temperature compared to the standard procedure for DOTATOC as model compound [26]. To take the advantage of additional ethanol, enhancing labeling yields,

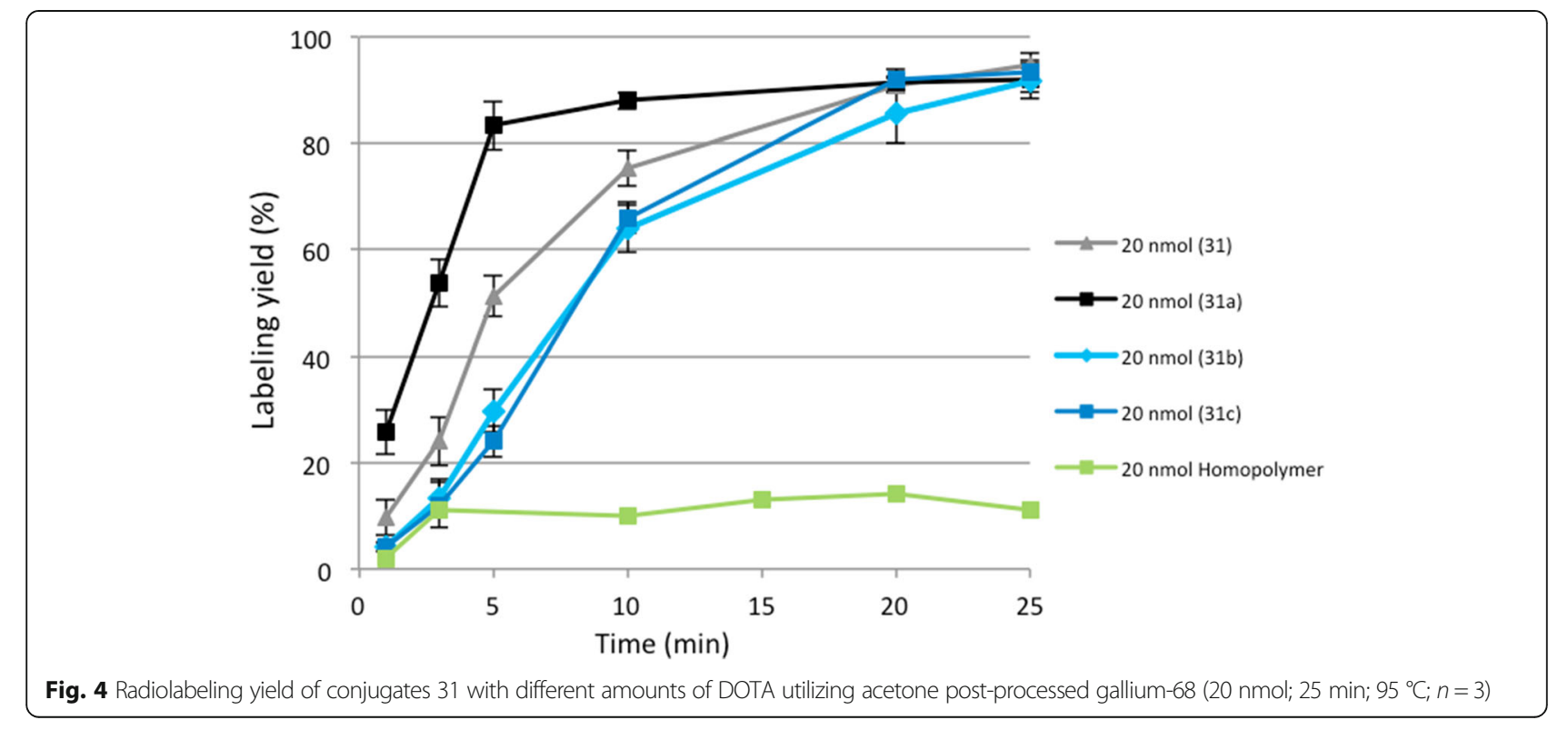




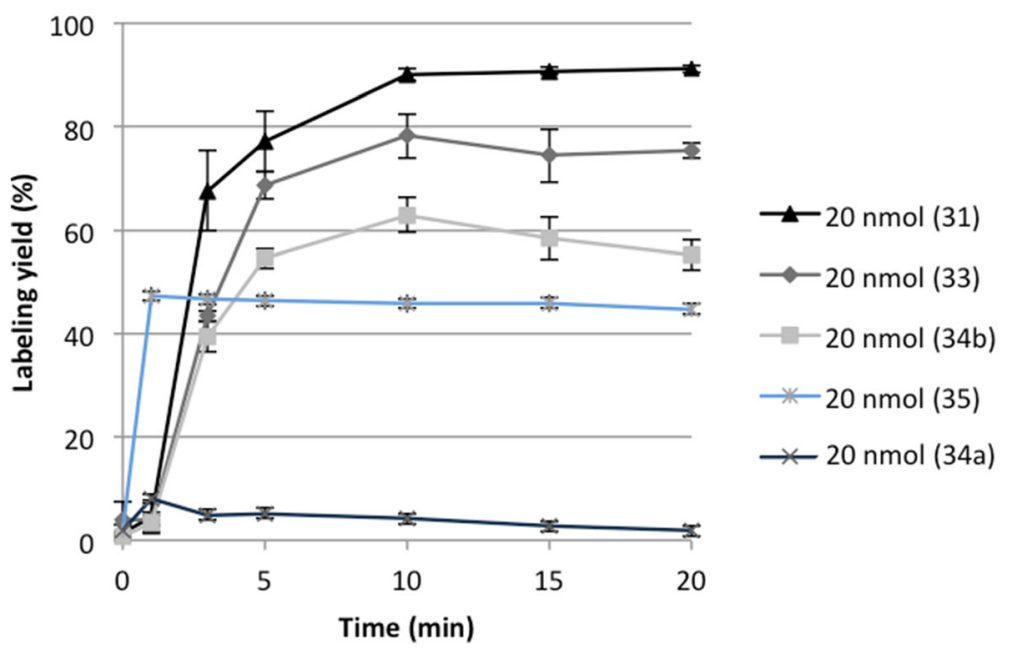

Fig. 5 Labeling yield using acetone post-processed gallium-68 (20 nmol; $\left.15 \mathrm{~min} ; 95^{\circ} \mathrm{C} ; n=3\right)$

conjugates 33-35 were labeled utilizing this protocol too. Additional file 1: Figure S1 shows the results of labeling conjugate 33 utilizing the two post-processing methods. Labeling with the ethanol post-processed gallium-68 in $1 \mathrm{M}$ NaHEPES buffer leads to significantly increased yields of complex formation. The difference between both labeling procedures is accounted for $20 \%$. An additional advantage of the ethanol-based post-processing is the possibility to reduce the overall volume of the labeling solution from $5.25 \mathrm{~mL}$ (acetone) to $2.25 \mathrm{~mL}$ (ethanol), and therefore to increase the specific activities.

This shows that labeling is also affected by the linker structure. Conjugates $\mathbf{3 3}$ and $\mathbf{3 5}$ have the same chain length $(n=11)$ but differ in their structure. Conjugate 33 contains a dodecyl linker while the positions 4 and 9 are substituted with oxygen atoms in the linker chain of $\mathbf{3 5}$. This structural difference is reflected in the decreasing labeling yield from $75 \% 33$ to $45 \% 35$.

\section{Purification of gallium-68-labeled DOTA-HPMA conjugates and quality control}

After labeling, the polymeric systems could be purified fast and easy using Sephadex G-25 size exclusion chromatography. Over $90 \%$ of the radiolabeled product can

Table 3 Labeling of HPMA-DOTA conjugates 31-35 using acetone and ethanol post-processed ${ }^{68} \mathrm{Ga}$ eluate $(20 \mathrm{nmol}$, $20 \min , 95^{\circ} \mathrm{C}$ )

\begin{tabular}{lllll}
\hline Polymer & Linker & \% DOTA & RCY acetone PP (\%) & RCY ethanol PP (\%) \\
\hline 31 & $(1)$ & 11 & $91 \pm 1$ & $98 \pm 1$ \\
33 & $(3)$ & 1.6 & $75 \pm 2$ & $97 \pm 1$ \\
$34 \mathrm{a}$ & $(4)$ & 0.6 & $2 \pm 1$ & $25 \pm 8$ \\
$34 \mathrm{~b}$ & $(4)$ & 1.2 & $55 \pm 3$ & $40 \pm 8$ \\
35 & $(5)$ & 3.5 & $45 \pm 22$ & $67 \pm 6$ \\
\hline
\end{tabular}

be collected in two fractions with radiochemical purity of $>98 \%$. As the product is eluted from the column with $0.9 \% \mathrm{NaCl}$, the resulting solution could be directly used for in vivo studies. Radiochemical analysis of complex formation was performed by TLC. The $R_{f}$ values for free uncomplexed gallium-68 and labeled conjugates were determined as $R_{f}\left(\left[{ }^{68} \mathrm{Ga}\right] \mathrm{Ga}^{3+}\right)=0.9$ and $R_{f}\left({ }^{68} \mathrm{Ga}\right.$ conjugate) $=0$. The $R_{f}$ values for scandium- 44 and lutetium177 utilizing the same method are the same as for gallium-68, 0.9.

\section{Radiolabeling with scandium-44}

As conjugate 31 showed the best radiolabeling yield, this conjugate was used for further labeling experiments with scandium-44 and lutetium-177. Scandium belongs to the transition metal group, and its complex formation strongly depends on $\mathrm{pH}$ of aqueous solution as previously shown for $\left[{ }^{44} \mathrm{Sc}\right]$ Sc-DOTATOC complex formation [35]. Utilizing the post-processing method leads to a final solution of scandium-44 in ammonium acetate buffer $(\mathrm{pH} 4)$ suitable for direct labelling [29]. Labeling with post-processed scandium-44 in ammonium acetate buffer ( $\mathrm{pH} 4$ ) yields up to $95 \%$ within 30 min for DOTA-HPMA 31 (Fig. 6).

\section{Radiolabeling with lutetium-177}

Radiolabeling with lutetium-177 is fast and efficient. Within 3 min, reaction is completed and yields up to $97 \%$ can be obtained for DOTA-conjugated HPMA 31 (Fig. 6).

\section{Stability of labeled compounds}

Stability of the formed complex is a crucial factor in the development of new radiopharmaceuticals. Therefore, several experiments were done to explore stability of the labeled conjugates in $\mathrm{NaCl}$ (used as final solvent), 


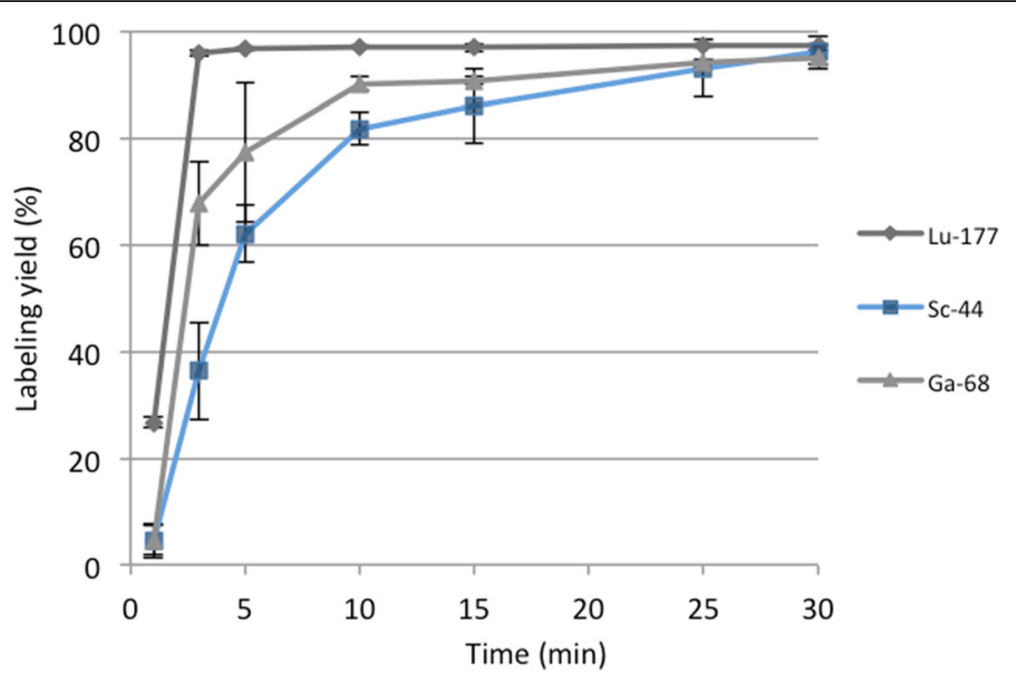

Fig. 6 Labeling yields for 31 with scandium-44, gallium-68, and lutetium-177 (20 nmol; 30 min; $\left.95^{\circ} \mathrm{C} ; n=3\right)$
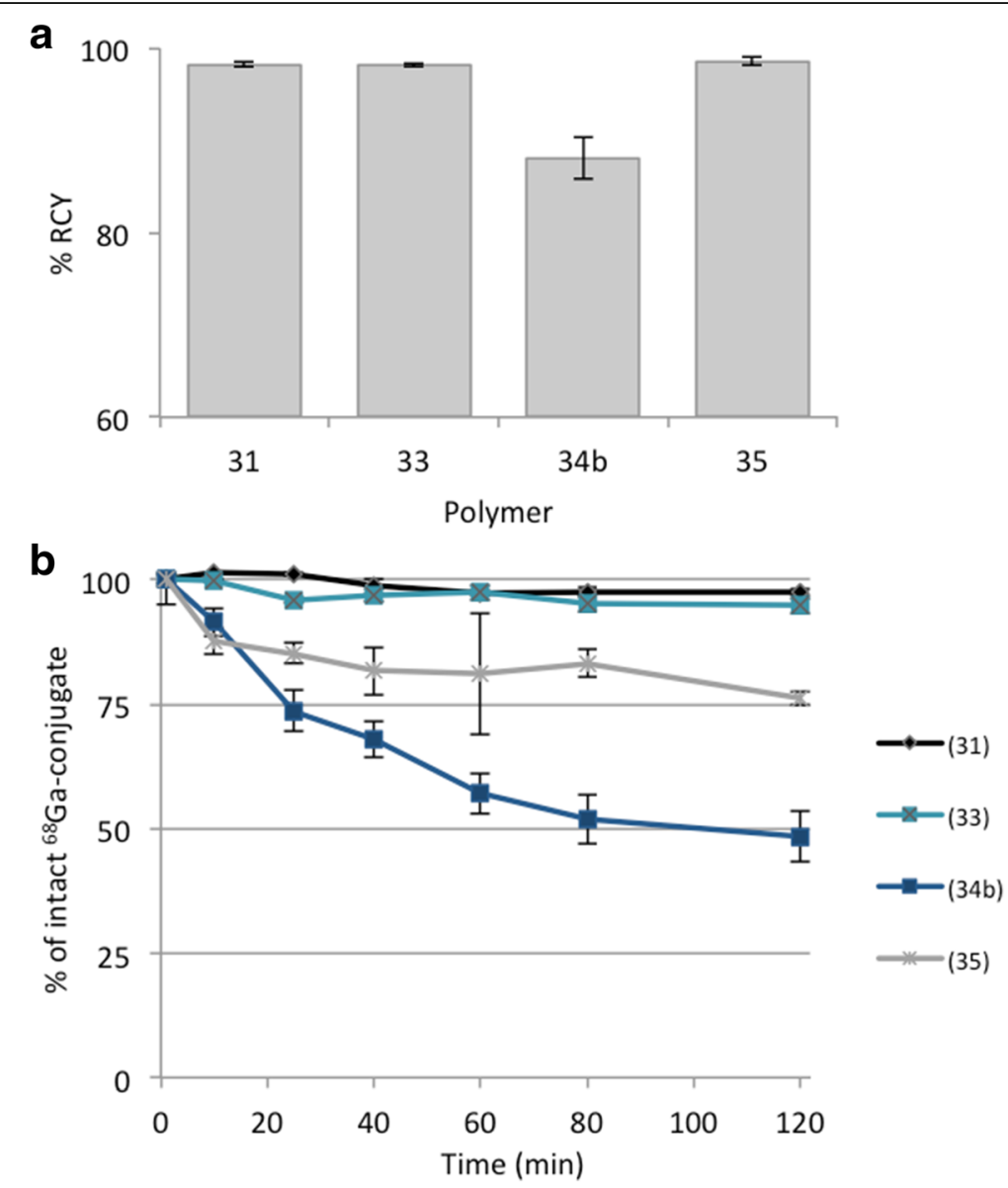

Fig. 7 Stability of gallium-68-labeled conjugates in $0.9 \% \mathrm{NaCl}(\mathbf{a})$ and in $\mathrm{HSA}(\mathbf{b})$ within 120 min at $37^{\circ} \mathrm{C}(n=3)$ 
human serum albumin, against trans-metalation $\left(\mathrm{Fe}^{3+}\right.$; $\mathrm{Ca}^{2+} ; \mathrm{Mg}^{2+}$ ) and trans-chelation (EDTA, DTPA).

Stability of gallium-68-labeled DOTA-HPMA conjugates in $0.9 \% \mathrm{NaCl}$ is presented in Fig. 7a. It indicates a high stability of the labeled conjugates even after 2 -h incubation at $37^{\circ} \mathrm{C}$.

In human serum albumin, the conjugates with alkyl linkers 31 and $\mathbf{3 3}$ indicate high stability. For both conjugates, the percentage of intact gallium- 68 conjugate is higher than $95 \%$. Contrary to that, the conjugates with alkoxy linker structure show higher decomposition rate (Fig. 7b).

The presence of different metal cations (e.g., $\mathrm{Fe}^{3+}$ ) in the final solution or in the blood can cause transmetalation of the radiolabeled conjugate and finally lead to a release of free radionuclide into solution. Therefore, it is necessary to determine whether the conjugate forms stable complexes in the presence of relevant metal cations or not prior to in vivo studies.

Conjugate 31c shows the highest stability against trans-metalation (Table 4). Similar to studies of 34-35 in HSA, stabilities are affected by addition of other metal cations. Within $120 \mathrm{~min}$, decomposition up to $30 \%$ can be observed. More complex are the results for 33. This conjugate indicates high stability in experiments with $\mathrm{HSA}, \mathrm{Fe}^{3+}$, and $\mathrm{Ca}^{2+}$ while decomposition is observed for challenge studies against $\mathrm{Mg}^{2+}$.

Similarly, stability against other complexing agents like EDTA and DTPA was investigated to check if transmetalation from HPMA-DOTA conjugate to competing ligand occurs. The results are shown in Table 5. Here, conjugates 34a and 34b show trans-metalation to DTPA with more than $30 \%$. All other conjugates are relatively stable to both agents.

Furthermore, one polymer (31c) was labeled with scandium-44 as described in Section 3.2.2, and its stability was evaluated in $0.9 \% \mathrm{NaCl}$ and HSA for up to $24 \mathrm{~h}$ at $37{ }^{\circ} \mathrm{C}$ in order to use this polymer for downstream in vivo applications. Table 6 shows that the ${ }^{44} \mathrm{Sc}$-labeled polymer is very stable over a time period of up to $24 \mathrm{~h}$ with values $>94 \%$ in $\mathrm{NaCl}$ and $\mathrm{HSA}$.

Table 4 Stability of gallium- 68 conjugates at $37^{\circ} \mathrm{C}$ in the presence of different metal cations at $10^{-2} \mathrm{M}$ concentrations $(n=3)$

\begin{tabular}{llllll}
\hline Conjugate & Linker & $\%$ DOTA & \multicolumn{3}{c}{$\%$ of intact ${ }^{68} \mathrm{Ga} \mathrm{conjugate} \pm \mathrm{SD}$} \\
\cline { 4 - 6 } & & & $\mathrm{Fe}^{3+}$ & $\mathrm{Mg}^{2+}$ & $\mathrm{Ca}^{2+}$ \\
\hline $31 \mathrm{c}$ & $(1)$ & 1.6 & $94 \pm 3$ & $92 \pm 1$ & $92 \pm 5$ \\
33 & $(3)$ & 1.6 & $94 \pm 9$ & $69 \pm 2$ & $99 \pm 2$ \\
$34 \mathrm{a}$ & $(4)$ & 0.6 & $88 \pm 2$ & $80 \pm 3$ & $70 \pm 1$ \\
$34 \mathrm{~b}$ & $(4)$ & 1.2 & $84 \pm 2$ & $86 \pm 2$ & $90 \pm 1$ \\
35 & $(5)$ & 3.5 & $74 \pm 2$ & $85 \pm 1$ & $83 \pm 8$ \\
\hline
\end{tabular}

Table 5 Stability of ${ }^{68} \mathrm{Ga}$ conjugates at $37^{\circ} \mathrm{C}$ in the presence of EDTA or DTPA at molar ratio 100:1 of competing ligand to conjugate $(n=3)$ after $120 \mathrm{~min}$

\begin{tabular}{lllll}
\hline Conjugate & Linker & $\%$ DOTA & \multicolumn{2}{l}{$\%$ of intact ${ }^{68} \mathrm{Ga}$ conjugate \pm SD } \\
\cline { 4 - 5 } & & & EDTA & DTPA \\
\hline $31 \mathrm{c}$ & $(1)$ & 1.6 & $92 \pm 5$ & $83 \pm 1$ \\
33 & $(3)$ & 1.6 & $90 \pm 2$ & $87 \pm 1$ \\
$34 \mathrm{a}$ & $(4)$ & 0.6 & $77 \pm 2$ & $59 \pm 1$ \\
$34 \mathrm{~b}$ & $(4)$ & 1.2 & $80 \pm 3$ & $61 \pm 1$ \\
35 & $(5)$ & 3.5 & $78 \pm 5$ & $93 \pm 1$ \\
\hline
\end{tabular}

\section{In vivo and ex vivo biodistribution}

For the biological evaluation, ${ }^{44} \mathrm{Sc}$-labeled derivatives were considered in order to demonstrate that the use of trivalent radiometals is favorable in extending the time period of molecular PET imaging compared to ${ }^{18} \mathrm{~F}$-labeled analogues without changing the pharmacokinetics. Because of the minimal alterations to structure and geometry and very good radiochemical purity and stability (Table 6), the ${ }^{44}$ Sc-labeled DOTA-HPMA polymer 31c with the lowest DOTA incorporation and a short alkyl linker was chosen and evaluated in B16 tumor-bearing mice. The dynamic PET acquisition over a time course of $2 \mathrm{~h}$ p.i. shows a fast renal excretion of the tracer (Fig. 8a) due to the low molecular weight of the HPMA homopolymer. The heart still shows a robust signal $2 \mathrm{~h}$ p.i. while the tumor hardly gets visible over time.

The pharmacokinetics over $24 \mathrm{~h}$ shows a relatively low but constant tumor uptake over $4 \mathrm{~h}$ and is still visible after $24 \mathrm{~h}$ (Fig. 8b) while the uptake in heart declines. The coronal slices show that the tracer mainly accumulates in the outer rim of the tumor after 4 and $24 \mathrm{~h}$. This hints at a poor vascularization inside the tumor what could also be observed during the dissection of the tumors.

These results are supported by the ex vivo evaluation of the different tissues (Fig. 9, Additional file 1: Table S1).

A fast clearance through the kidneys is observed after $2 \mathrm{~h}$ with values of $32.9 \pm 6.6 \% \mathrm{ID} / \mathrm{g}$ (urine: $54.6 \pm 9.2 \%$ $\mathrm{ID} / \mathrm{g}$ ) staying constantly over time. After $24 \mathrm{~h}$, the activity in urine dropped to $32.4 \pm 10.2 \% \mathrm{ID} / \mathrm{g}$.

Uptake in the lungs, liver, and heart remained considerably constant during the three time points studied

Table 6 Stability of ${ }^{44} \mathrm{Sc}$ conjugate $31 \mathrm{C}$ at $37^{\circ} \mathrm{C}$ in $0.9 \% \mathrm{NaCl}$ and HSA during $24 \mathrm{~h}(n=3)$

\begin{tabular}{lll}
\hline Time $(h)$ & \multicolumn{2}{l}{$\%$ of intact ${ }^{44}$ Sc conjugate \pm SD } \\
\cline { 2 - 3 } & $0.9 \% \mathrm{NaCl}$ & HSA \\
\hline 1 & $98.7 \pm 0.5$ & $96.7 \pm 0.9$ \\
4 & $98.3 \pm 0.9$ & $95.7 \pm 0.5$ \\
24 & $97.7 \pm 0.5$ & $94.3 \pm 0.5$ \\
\hline
\end{tabular}


a
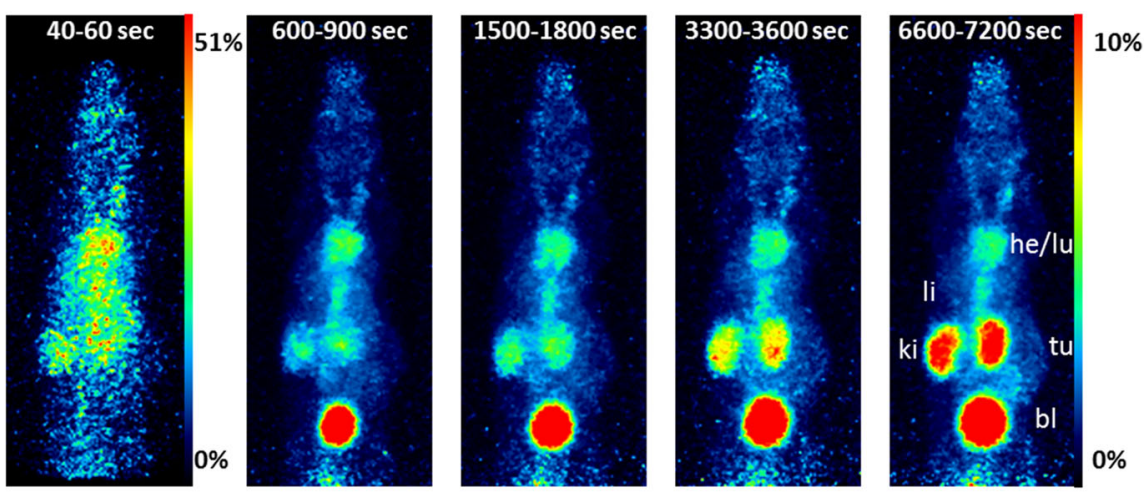

b

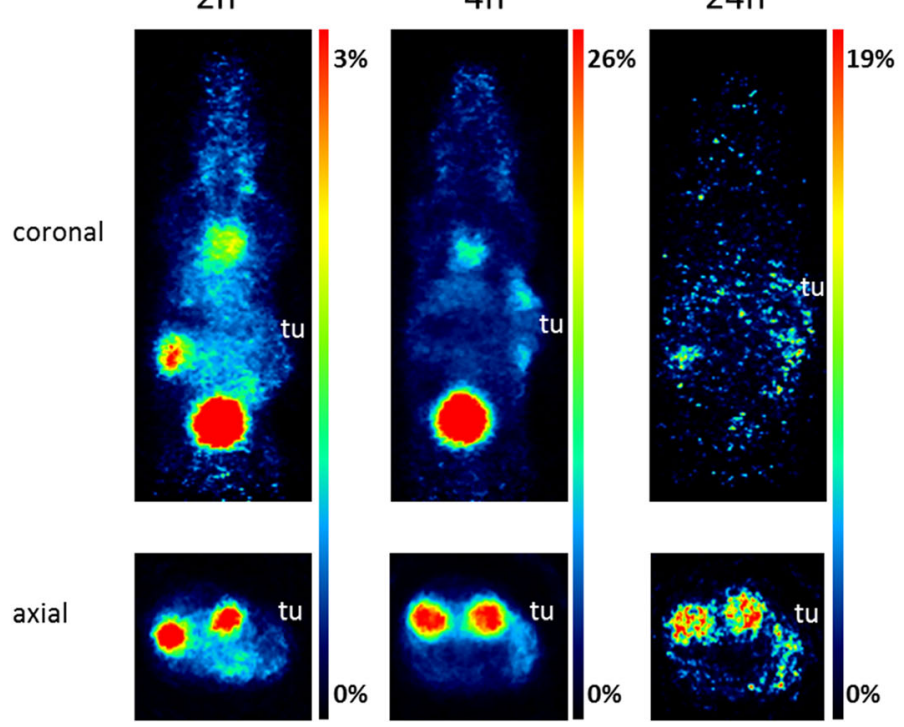

Fig. 8 a In vivo dynamic PET of $\left[{ }^{44}\right.$ Sc]Sc-DOTA-HPMA 31c in a tumor-bearing mouse $(n=1)$. Maximum intensity projections (MIPs) of different time points during 2-h acquisition. b Representative static PET images of $\left.{ }^{44} \mathrm{Sc}\right] \mathrm{Sc}$-DOTA-HPMA 31c in tumor-bearing mice 2,4 , and $24 \mathrm{~h}$ p.i. (the last $30 \mathrm{~min}$ is imaged). Coronal and axial slices are shown with focus on tumor

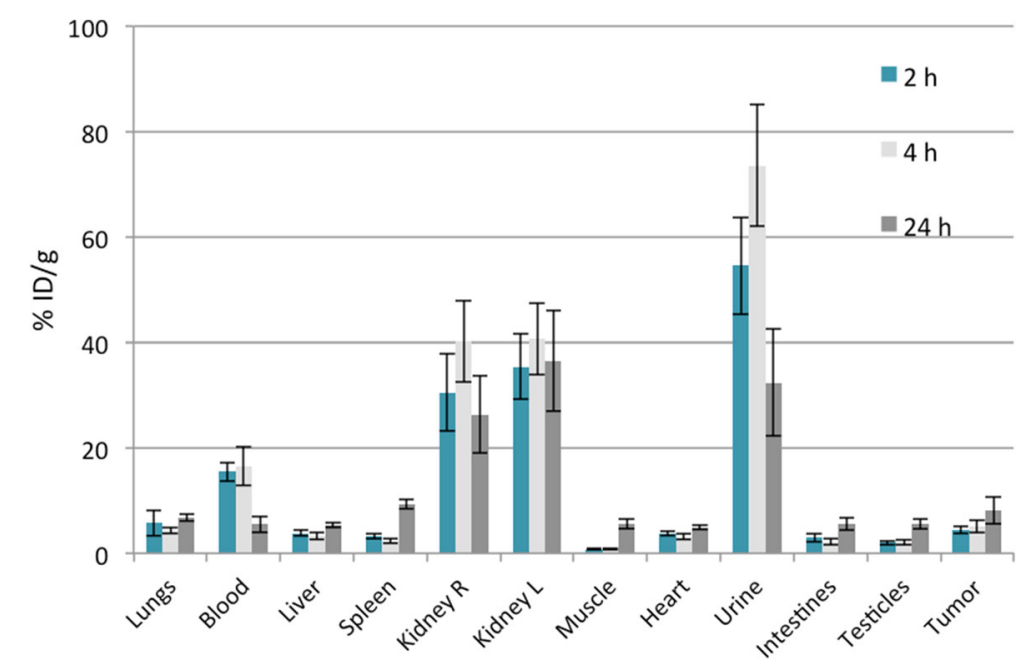

Fig. 9 Ex vivo biodistribution of $\left[{ }^{44}\right.$ Sc]Sc-DOTA-HPMA 31c in tumor-bearing mice ( $n=5$ for each time point) 
with mean values of $5.6 \pm 1.2,4.2 \pm 0.5$, and $4.0 \pm 0.5 \%$ $\mathrm{ID} / \mathrm{g}$ (mean values for each organ over time) respectively. Uptake in the spleen increased after $24 \mathrm{~h}$ when compared to the earlier time points. The tumor uptake after $2 \mathrm{~h}$ p.i. was of $4.4 \pm 0.8 \% \mathrm{ID} / \mathrm{g}$ and slightly increased to $5.1 \pm 1.7 \%$ ID/g after $4 \mathrm{~h}$ p.i. The blood values are comparable between $2 \mathrm{~h}(15.5 \pm 1.8 \% \mathrm{ID} / \mathrm{g})$ and $4 \mathrm{~h}$ p.i $(16.6 \pm 3.7 \%$ $\mathrm{ID} / \mathrm{g})$. Interestingly, the tumor uptake further increases after $24 \mathrm{~h}$ (to $8.2 \pm 3.1 \% \mathrm{ID} / \mathrm{g}$ ) while tracer concentration in the blood decreases $(5.5 \pm 1.5 \% \mathrm{ID} / \mathrm{g})$.

Taken together, ${ }^{44} \mathrm{Sc}$-DOTA labeling of HPMA homopolymers is well suited for measuring the body distribution for a time period of up to $24 \mathrm{~h}$ in vivo and ex vivo.

\section{Ex vivo metabolism}

In order to investigate the in vivo stability of the labeled polymer with scandium-44, the blood samples obtained during biodistribution were separated into different fractions: blood cells, polymer + proteins, and plasma water. Figure 10 shows the amount of radioactivity in the different fractions with reference to the total amount of radioactivity in the blood samples of ${ }^{44} \mathrm{Sc}$ conjugate 31c.

The fraction containing proteins and protein-bound polymer presents $55.2 \pm 1.4 \%$ of total activity in blood $2 \mathrm{~h}$ p.i. This value remained stable after 4 and $24 \mathrm{~h}$ p.i. with values of $49.8 \pm 6.6 \%$ and $52.1 \pm 6.3 \%$ respectively. In the blood cell fractions, the activity also remained constant during the three time points analyzed (values ranged from $37.2 \pm 2.2 \%$ to $40.1 \pm 1.3 \%$ ). Only a small proportion of about $10 \%$ was found in the plasma water comprising of free scandium-44 and maybe a small amount of $\left[{ }^{44} \mathrm{Sc}\right] \mathrm{Sc}$-HPMA that could not be precipitated completely indicating a good in vivo stability.

\section{Discussion}

Optimized conjugation of DOTA onto HPMA backbone yielded several DOTA-linker-poly(HPMA) conjugates with different linker structures and with variation of amount of DOTA. These derivatives were labeled with various metallic radionuclides (gallium-68, scandium-44, lutetium-177). Labeling and stability of the produced conjugates were investigated in detail with the generatorderived PET radionuclide gallium- 68 .

It was observed that labeling kinetics with gallium-68 slightly depend on the incorporation rate of DOTA. There seems to be a kind of an optimum amount of 4.8\% DOTA for a very fast labeling and at the same time $11 \%$ DOTA is too much to efficiently coordinate gallium into the chelator and 1.6\% DOTA is insufficient to quantitatively bind gallium, but after 25 min, no differences between the various DOTA amounts were evident. This shows that a minimum of $1.6 \%$ DOTA is enough to obtain labeling yields higher than $90 \%$. The linker structure also is a variable that influences labeling efficiency. Alkoxy linker structure generally exhibits lower labeling yields than alkane linkers despite of similar chain length and DOTA incorporation. Moreover, it was observed that a short linker (two-carbon chain) is enough to provide quantitative radiolabeling yields. Another investigated effect is the radiolabeling in the presence of ethanol, which is known to improve labeling efficacy.

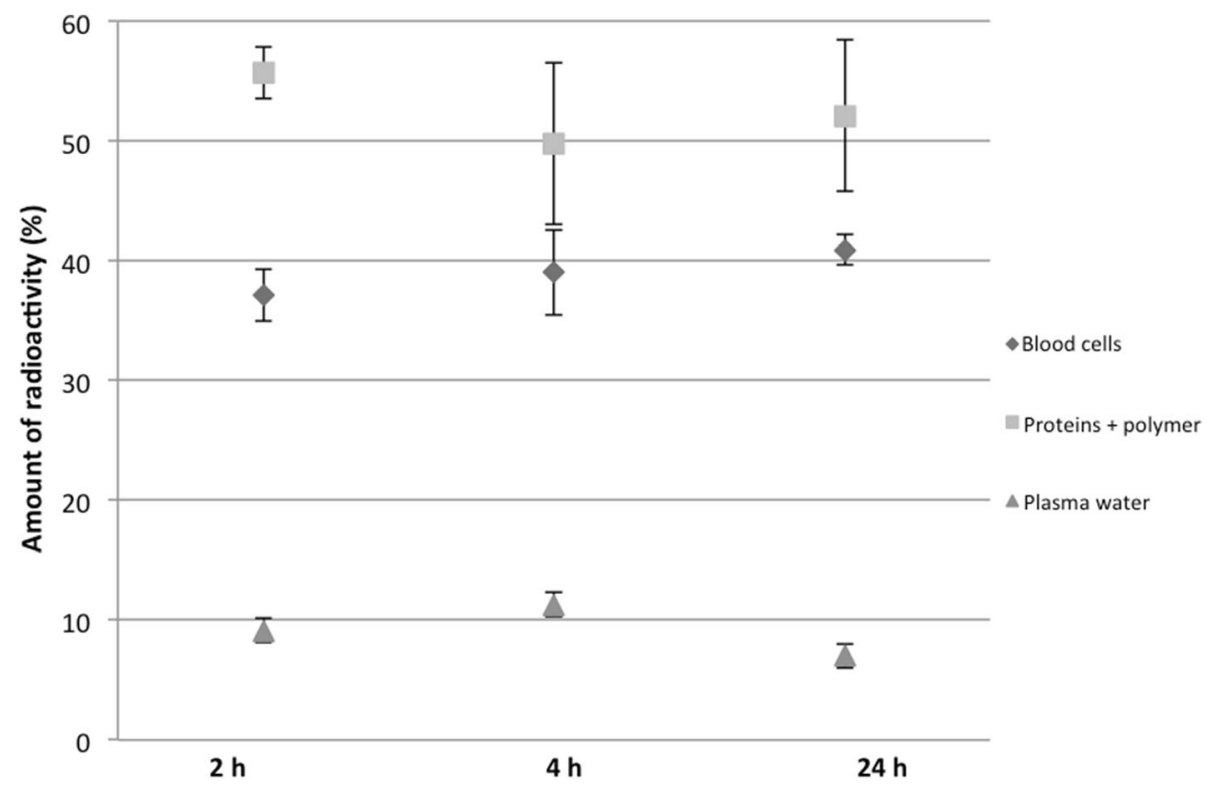

Fig. 10 Distribution of $\left.{ }^{44} \mathrm{Sc}\right] \mathrm{Sc}-31 \mathrm{c}$ in the blood for up to $24 \mathrm{~h}(n=3)$. Data is expressed as percentage of the amount of radioactivity that was present in the whole blood sample (mean \pm SEM) 
Utilizing the ethanol post-processed ${ }^{68} \mathrm{Ga}$ eluate enhances the labeling efficacy for all conjugates. In conclusion, ca. $1.6 \%$ of DOTA per HPMA polymer allow achieving quantitative radiolabeling, if the optimal conditions are applied.

With regard to future in vivo evaluations, special efforts were focused on stability studies of ${ }^{68} \mathrm{Ga}$-labeled conjugates, to adopt the successful candidate to scandium-44 (PET imaging) and lutetium-177 (therapy). Only the conjugates with alkyl chains as linker structure provide high stability in all examined solutions: $0.9 \% \mathrm{NaCl}$, HSA, metal cations $\left(\mathrm{Fe}^{3+}, \mathrm{Mg}^{2+}, \mathrm{Ca}^{2+}\right)$, and competing ligands (EDTA, DTPA). In general, alkoxy linkers show lower labeling yields and stability than alkyl linker conjugates irrespective of DOTA content. Within the group of conjugates containing alkyl linkers, DOTA content seems to have a mild impact on labeling yield because there is a little difference between the conjugate containing 11 and 1.6\% DOTA. In the conjugates containing alkoxy linkers, there might be a kind of threshold for DOTA concerning labeling yield. At $0.6 \%$, almost no labeling could be achieved whereas with $1.2 \%$, high labeling yields were evident. Furthermore, the structure of the linker has more influence on labeling yield and stability than the linker length. That can be explained as a possible unstable complexation of $\mathrm{Ga}$ (III) with the oxygen present in the linker. Our hypothesis is that the oxygens on the HPMA structure could induce a more aggregated structure with alkoxy linkers compared to those without oxygens, maybe by van der Waals interaction as it was shown for other biopolymers [37], thus hindering the DOTA availability for the metals. Furthermore, there is no competition between the oxygens and the DOTA. More likely, the van der Waals interactions slightly reduce the complexation of DOTA with the metal.

The conjugate 31c was also labeled with scandium-44 and further analyzed in vivo because of the minimal alterations to structure and geometry and very good radiochemical purity and stability. The polymer shows a fast renal clearance in vivo.

These findings of the in vivo measured tracer kinetics are in line with earlier data on low molecular weight HPMA homopolymers, which were labeled with fluorine18 and showed comparable pharmacokinetics after $2 \mathrm{~h}$ [20]. By labeling with the longer-lived scandium-44, imaging time could be prolonged which favors imaging of slower molecular processes like the accumulation of targeted drugs into tumors or the activation of $\mathrm{T}$ cells after a special vaccination.

HPMA-copolymer conjugates have been successfully utilized in the delivery of miscellaneous drugs (e.g., doxorubicin [14], paclitaxel [15], camptothecin [16]). Modification can be achieved through activated comonomers leading to biocompatible polymers with functionalities for polymer-analogue reaction. This strategy is very flexible in the choice of ligands, type of activation, and occupancy rate and was already used in various studies [17]. Reaction of a reactive ester polymer with several ligands leads to multifunctionalized copolymers which can be applied for theranostic applications. Recently, a synthetic pathway was shown that combines controlled radical polymerization with post-polymerization modification of a perfluorinated reactive ester [38] to get access to well-defined HPMA-based block copolymers of low dispersity and controlled copolymer structure with a high degree of multiple functionalities. With this technology, advanced HPMA-based polymer conjugates and nano-sized self-assemblies could be developed that may serve as novel drug carriers. Moreover, the high degree of functionalities enables novel applications for HPMAbased copolymers, for instance, in the field of tumor immunotherapy [39].

Interestingly, despite the low molecular weight, without any expected EPR effect, tumor accumulation slightly increased over time while tracer concentration in the blood decreased. The enhanced tumor accumulation could be due to the higher permeability reported for B16F10 tumors compared to any other tumor types [40]. However, this 24-h blood value is also well comparable to earlier data by Lammers et al. [41] were they labeled HPMA copolymers of different weights by iodination (iodine-131) and discovered the body distribution up to $168 \mathrm{~h}$ p.i. [41]. Here, the smallest evaluated polymer $(23 \mathrm{kDa})$ showed a similar blood retention after $24 \mathrm{~h}$ like the HPMA polymer in our study even though those studies were performed in rats and with another tumor model.

Interestingly, our findings on the different blood components suggested that the homopolymer interacts with serum proteins and with blood cells. Former studies of our group showed that HPMA-ran-LMA copolymers interact with very low density lipoproteins in the blood [42] and with cell membranes [43, 44]. Not only structure and size of the polymer but furthermore the cell type is crucial for polymer delivery. Moreover, hydrophilic homopolymers (like in our case) were taken up by micropinocytosis as well as by clathrin-mediated and clathrin-independent endocytosis [45].

Furthermore, it is well known that nanoparticles and polymers are opsonized by plasma proteins upon entering the blood stream $[46,47]$ which alters their molecular size, charge, and aggregation behavior and thereby their physicochemical properties dramatically. One could imagine that the HPMA polymers used in this study get "sticky" by the protein corona and may bind unspecifically to cells in the blood stream (monocytes, granulocytes, $\mathrm{B}$ and $\mathrm{T}$ cells) or may even be endocytosed. Especially, when polymers are opsonized by complement factors, which were also identified in the protein corona 
of nanocarriers $[48,49]$, they will be taken up rapidly by circulating cells of the immune system. Several in vitro studies showed that dendritic cells can bind and take up HPMA-based homopolymers [39, 50] and even other immune cells like granulocytes and $\mathrm{B}$ and $\mathrm{T}$ cells do so.

To our knowledge, our study is the first proof of principle study that analyzes the labeling of HMPA polymers with scandium-44 and its in vivo distribution. Besides high in vitro stabilities, the good in vivo stability enables imaging of biological processes for up to $24 \mathrm{~h}$. Further studies will focus on the comparison of different molecular weight polymers for enhanced EPR-mediated enrichment into tumors as well as receptor-mediated targeting to tumors as it was shown for $\left[{ }^{44} \mathrm{Sc}\right] \mathrm{Sc}$-DOTANAPamide for melanoma imaging [51].

\section{Conclusions}

This study confirms the principle applicability of HPMA conjugates for labeling with different metallic radionuclides with established radiolabeling chemistry. Introducing as less than only $1.6 \%$ of DOTA chelator using a short linker chain, which facilitates the synthesis and efficiency of the polymer conjugates, allows introduction of both diagnostic and therapeutic radiometals. The introduction of $\left[{ }^{44} \mathrm{Sc}\right] \mathrm{Sc}$-DOTA as well as lutetium-177 into HPMA polymers allows for longer observation times than for example ${ }^{18} \mathrm{~F}$-labeling being a good prerequisite for tumor imaging and therapy in the future.

\section{Additional file}

Additional file 1: Supporting information. (ZIP $77 \mathrm{~kb}$ )

\section{Abbreviations}

DOTA: 1,4,7,10-Tetraazacyclododecane-1,4,7,10-tetraacetic acid; DTPA: Diethylenetriaminepentaacetic acid; EDTA: Ethylenediaminetetraacetic acid; EPR: Enhanced permeability and retention; GPC: Gel permeation chromatography; HPMA: N-(2-Hydroxypropyl)methacrylamide; HSA: Human serum albumin; $\mathrm{NaCl}$ : Sodium chloride; NMR: Nuclear magnetic resonance; PDI: Polydispersity index; PET: Positron emission tomography;

THF: Tetrahydrofuran; TLC: Thin-layer chromatography

\section{Acknowledgements}

The authors would like to thank Nicole Bausbacher for her support during the animal studies.

\section{Funding}

E. Eppard has been funded by the Dr. Georg-Scheuing Stiftung. A. de la Fuente has been funded by the People Programme (Marie Curie Actions) of the European Union's Seventh Framework Programme (FP7/2007-2013) under REA grant agreement no. PITN-GA-2012-317019 "Trace 'n Treat". All other authors have been funded by the DFG (SFB1066).

\section{Availability of data and materials}

The datasets supporting the conclusions of this article are included within the article (and in Additional file 1).

\section{Authors' contributions}

EE and AdIF performed the experiments, analyzed the data, and wrote the manuscript. MA and NM performed the experiments and analyzed the data.
SP designed and performed the animal studies, analyzed the data, and wrote the manuscript. MM, RZ, and FR designed the study and wrote the manuscript. All authors read and approved the final manuscript.

\section{Ethics approval and consent to participate}

All experiments were performed in accordance with federal guidelines and approved by the ethical committee of the state of Rhineland-Palatinate (according to §8 Abs. 1 Protection of Animals Act, Regional Investigational Authority Institute/Office Landesuntersuchungsamt; permission no. 23177-07/ G12-1-092 to M. Miederer).

\section{Consent for publication}

Not applicable.

\section{Competing interests}

The authors declare that they have no competing interests.

\section{Publisher's Note}

Springer Nature remains neutral with regard to jurisdictional claims in published maps and institutional affiliations.

\section{Author details}

${ }^{1}$ Clinic for Nuclear Medicine, University Medical Center Bonn, Bonn, Germany. ${ }^{2}$ Institute of Nuclear Chemistry, Johannes Gutenberg University Mainz, Mainz, Germany. ${ }^{3}$ Institute of Organic Chemistry, Johannes Gutenberg University Mainz, Mainz, Germany. ${ }^{4}$ Clinic for Nuclear Medicine, University Medical Center Mainz, Langenbeckstraße 1, 55131 Mainz, Germany.

Received: 8 December 2017 Accepted: 19 February 2018

Published online: 27 February 2018

\section{References}

1. Arranja AG, Pathak V, Lammers T, Shi Y. Tumor-targeted nanomedicines for cancer theranostics. Pharmacol Res. 2017;115:87-95.

2. Landesman-Milo D, Peer D. Transforming nanomedicines from lab scale production to novel clinical modality. Bioconjug Chem. 2016;27(4):855-62.

3. Iyer AK, Khaled G, Fang J, Maeda H. Exploiting the enhanced permeability and retention effect for tumor targeting. Drug Discov Today. 2006;11(17-18):812-8.

4. Maeda $\mathrm{H}$, Nakamura $\mathrm{H}$, Fang J. The EPR effect for macromolecular drug delivery to solid tumors: improvement of tumor uptake, lowering of systemic toxicity, and distinct tumor imaging in vivo. Adv Drug Deliv Rev. 2013;65(1):71-9.

5. Noguchi Y, Wu J, Duncan R, Strohalm J, Ulbrich K, Akaike T, et al. Early phase tumor accumulation of macromolecules: a great difference in clearance rate between tumor and normal tissues. Jpn J Cancer Res. 1998; 89(3):307-14.

6. Kopeček J, Baẑilová H. Poly[N-(2-hydroxypropyl)methacrylamide]-I. Radical polymerization and copolymerization. Eur Polym J. 1973;9(1):7-14.

7. Bohdanecky M, Baẑilová H, Kopeček J. Poly[N-(2hydroxypropyl)methacrylamide] —II: hydrodynamic properties of dilute solutions. Eur Polym J. 1974;10(5):405-10.

8. Kopecek J, Sprincl L. Relationship between the structure and biocompatibility of hydrophilic gels. Polim Med. 1974;4(2):109-17.

9. Kopecek J, Sprincl L, Bazilova H, Vacik J. Biological tolerance of poly(Nsubstituted acrylamides). J Biomed Mater Res. 1973;7(1):111-21.

10. Kopecek J, Sprincl L, Lim D. New types of synthetic infusion solutions. I. Investigation of the effect of solutions of some hydrophilic polymers on blood. J Biomed Mater Res. 1973;7(2):179-91.

11. Sprincl L, Kopecek J, Lim D. Effect of porosity of heterogeneous poly(glycol monomethacrylate) gels on the healing-in of test implants. J Biomed Mater Res. 1971;5(5):447-58.

12. Sprincl L, Vacik J, Kopecek J. Biological tolerance of ionogenic hydrophilic gels. J Biomed Mater Res. 1973;7(1):123-36.

13. Ulbrich K, Sprincl L, Kopecek J. Biocompatibility of poly (2,4-pentadiene-1ol). J Biomed Mater Res. 1974;8(2):155-61.

14. Vasey PA, Kaye SB, Morrison R, Twelves C, Wilson P, Duncan R, et al. Phase I clinical and pharmacokinetic study of PK1 [N-(2-

hydroxypropyl)methacrylamide copolymer doxorubicin]: first member of a new class of chemotherapeutic agents-drug-polymer conjugates. Cancer Research Campaign Phase I/II Committee. Clin Cancer Res. 1999;5(1):83-94. 
15. Meerum Terwogt JM, ten Bokkel Huinink WW, Schellens JH, Schot M, Mandjes IA, Zurlo MG, et al. Phase I clinical and pharmacokinetic study of PNU166945, a novel water-soluble polymer-conjugated prodrug of paclitaxel. Anti-Cancer Drugs. 2001;12(4):315-23.

16. Schoemaker NE, van Kesteren C, Rosing H, Jansen S, Swart M, Lieverst J, et al. A phase I and pharmacokinetic study of MAG-CPT, a water-soluble polymer conjugate of camptothecin. Br J Cancer. 2002;87(6):608-14.

17. Kopecek J, Kopeckova P, Minko T, Lu Z. HPMA copolymer-anticancer drug conjugates: design, activity, and mechanism of action. Eur J Pharm Biopharm. 2000;50(1):61-81.

18. Sprincl L, Kopecek J, Vacik J, Lim D. Biological tolerance of poly(Nsubstituted methacrylamides). J Biomed Mater Res. 1971;5(3):197-205.

19. Chidambaram M, Manavalan R, Kathiresan K. Nanotherapeutics to overcome conventional cancer chemotherapy limitations. J Pharm Pharm Sci. 2011; 14(1):67-77.

20. Allmeroth M, Moderegger D, Biesalski B, Koynov K, Rosch F, Thews O, et al. Modifying the body distribution of HPMA-based copolymers by molecular weight and aggregate formation. Biomacromolecules. 2011;12(7):2841-9.

21. Allmeroth M, Moderegger D, Gundel D, Buchholz HG, Mohr N, Koynov K, et al. PEGylation of HPMA-based block copolymers enhances tumor accumulation in vivo: a quantitative study using radiolabeling and positron emission tomography. J Control Release. 2013;172(1):77-85.

22. Breeman WA, de Jong $M$, de Blois $E$, Bernard BF, Konijnenberg M, Krenning EP. Radiolabelling DOTA-peptides with 68Ga. Eur J Nucl Med Mol Imaging. 2005;32(4):478-85

23. Mueller D, Klette I, Baum RP, Gottschaldt M, Schultz MK, Breeman WA. Simplified $\mathrm{NaCl}$ based (68) Ga concentration and labeling procedure for rapid synthesis of (68)Ga radiopharmaceuticals in high radiochemical purity. Bioconjug Chem. 2012;23(8):1712-7.

24. Meyer GJ, Macke H, Schuhmacher J, Knapp WH, Hofmann M. 68Ga-labelled DOTA-derivatised peptide ligands. Eur J Nucl Med Mol Imaging. 2004;31(8): 1097-104.

25. Zhernosekov KP, Filosofov DV, Baum RP, Aschoff P, Bihl H, Razbash AA, et al. Processing of generator-produced 68Ga for medical application. J Nucl Med. 2007;48(10):1741-8

26. Eppard E, Wuttke M, Nicodemus PL, Rosch F. Ethanol-based post-processing of generator-derived (6)(8)Ga toward kit-type preparation of (6)(8)Garadiopharmaceuticals. J Nucl Med. 2014;55(6):1023-8.

27. Rosch F, Baum RP. Generator-based PET radiopharmaceuticals for molecular imaging of tumours: on the way to THERANOSTICS. Dalton Trans. 2011; 40(23):6104-11.

28. Filosofov DV, Loktionova NS, Rösch F. A 44Ti/44Sc radionuclide generator for potential application of 44Sc-based PET-radiopharmaceuticals. Radiochimica Acta Int J Chem Aspects Nucl Sci Technol. 2010;98:149-56.

29. Pruszynski M, Loktionova NS, Filosofov DV, Rosch F. Post-elution processing of (44)Ti/(44)Sc generator-derived (44)Sc for clinical application. Appl Radiat Isot. 2010;68(9):1636-41.

30. Liu Z, Ma T, Liu H, Jin Z, Sun X, Zhao H, et al. 177Lu-labeled antibodies for EGFR-targeted SPECT/CT imaging and radioimmunotherapy in a preclinical head and neck carcinoma model. Mol Pharm. 2014;11(3):800-7.

31. Seymour LW, Ferry DR, Anderson D, Hesslewood S, Julyan PJ, Poyner R, et al. Hepatic drug targeting: phase I evaluation of polymer-bound doxorubicin. J Clin Oncol. 2002;20(6):1668-76.

32. Mitra A, Nan A, Ghandehari H, McNeill E, Mulholland J, Line BR. Technetium99m-labeled N-(2-hydroxypropyl) methacrylamide copolymers: synthesis, characterization, and in vivo biodistribution. Pharm Res. 2004;21(7):1153-9.

33. Benezra M, Penate-Medina O, Zanzonico PB, Schaer D, Ow H, Burns A, et al. Multimodal silica nanoparticles are effective cancer-targeted probes in a model of human melanoma. J Clin Invest. 2011;121(7):2768-80.

34. Mohr N, Barz M, Forst R, Zentel R. A deeper insight into the postpolymerization modification of polypenta fluorophenyl methacrylates to poly(n-(2-hydroxypropyl) methacrylamide). Macromol Rapid Commun. 2014;35(17):1522-7.

35. Pruszynski M, Majkowska-Pilip A, Loktionova NS, Eppard E, Roesch F. Radiolabeling of DOTATOC with the long-lived positron emitter 44Sc. Appl Radiat Isot. 2012;70(6):974-9.

36. Roesch F, Perez-Malo CM. Improved efficacy of synthesis of $68 \mathrm{Ga}$ radiopharmaceuticals in mixtures of aqueous solution and non-aqueous solvents. J Nucl Med. 2013;54(supplement 2):163.
37. Van Oss CJ, Good RJ, Chaudhury MK. The role of van der Waals forces and hydrogen bonds in "hydrophobic interactions" between biopolymers and low energy surfaces. J Colloid Interface Sci. 1986;111(2):378-90.

38. Nuhn L, Barz M, Zentel R. New perspectives of HPMA-based copolymers derived by post-polymerization modification. Macromol Biosci. 2014;14(5): 607-18.

39. Mohr N, Kappel C, Kramer S, Bros M, Grabbe S, Zentel R. Targeting cells of the immune system: mannosylated HPMA-LMA block-copolymer micelles for targeting of dendritic cells. Nanomedicine (London, England). 2016; 11(20):2679-97.

40. Mei KC, Bai J, Lorrio S, Wang JT, Al-Jamal KT. Investigating the effect of tumor vascularization on magnetic targeting in vivo using retrospective design of experiment. Biomaterials. 2016;106:276-85.

41. Lammers T, Kuhnlein R, Kissel M, Subr V, Etrych T, Pola R, et al. Effect of physicochemical modification on the biodistribution and tumor accumulation of HPMA copolymers. J Control Release. 2005;110(1):103-18.

42. Hemmelmann M, Mohr K, Fischer K, Zentel R, Schmidt M. Interaction of pHPMA-pLMA copolymers with human blood serum and its components. Mol Pharm. 2013;10(10):3769-75.

43. Clemens-Hemmelmann M, Kuffner C, Metz V, Kircher L, Schmitt U, Hiemke

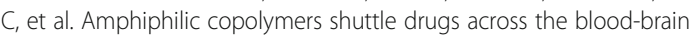
barrier. Macromol Biosci. 2016:16(5):655-65.

44. Hemmelmann M, Metz W, Koynov K, Blank K, Postina R, Zentel R. Amphiphilic HPMA-LMA copolymers increase the transport of rhodamine 123 across a BBB model without harming its barrier integrity. J Control Release. 2012;163(2):170-7.

45. Gundel D, Allmeroth M, Reime S, Zentel R, Thews O. Endocytotic uptake of HPMA-based polymers by different cancer cells: impact of extracellular acidosis and hypoxia. Int J Nanomedicine. 2017;12:5571-84.

46. Walczyk D, Bombelli FB, Monopoli MP, Lynch I, Dawson KA. What the cell "sees" in bionanoscience. J Am Chem Soc. 2010;132(16):5761-8.

47. Tenzer S, Docter D, Kuharev J, Musyanovych A, Fetz V, Hecht R, et al. Rapid formation of plasma protein corona critically affects nanoparticle pathophysiology. Nat Nanotechnol. 2013;8(10):772-81.

48. Tenzer S, Docter D, Rosfa S, Wlodarski A, Kuharev J, Rekik A, et al. Nanoparticle size is a critical physicochemical determinant of the human blood plasma corona: a comprehensive quantitative proteomic analysis. ACS Nano. 2011;5(9):7155-67.

49. Allemann E, Gravel P, Leroux JC, Balant L, Gurny R. Kinetics of blood component adsorption on poly(D,L-lactic acid) nanoparticles: evidence of complement C3 component involvement. J Biomed Mater Res. 1997;37(2): 229-34.

50. Tappertzhofen K, Bednarczyk M, Koynov K, Bros M, Grabbe S, Zentel R. Toward anticancer Immunotherapeutics: well-defined polymer-antibody conjugates for selective dendritic cell targeting. Macromol Biosci. 2014; 14(10):1444-57.

51. Nagy G, Denes N, Kis A, Szabo JP, Berenyi E, Garai I, et al. Preclinical evaluation of melanocortin-1 receptor (MC1-R) specific 68Ga- and 44Sc-labeled DOTANAPamide in melanoma imaging. Eur J Pharm Sci. 2017;106:336-44.

\section{Submit your manuscript to a SpringerOpen ${ }^{\circ}$ journal and benefit from:}

- Convenient online submission

- Rigorous peer review

- Open access: articles freely available online

- High visibility within the field

Retaining the copyright to your article

Submit your next manuscript at springeropen.com 